\title{
Protective and Harmful Effects of Physical Activity for Low Back Pain: A Protocol for the AUstralian Twin BACK Pain (AUTBACK) Feasibility Study
}

\author{
Marina B. Pinheiro, ${ }^{1}$ Manuela L. Ferreira, ${ }^{2}$ Kathryn Refshauge, ${ }^{1}$ John Hopper, ${ }^{3}$ Christopher G. Maher, ${ }^{4}$ \\ Jan Hartvigsen, ${ }^{5}$ Bart Koes, $^{6}$ Markus Hübscher, ${ }^{7}$ and Paulo H. Ferreira ${ }^{1}$ \\ ${ }^{1}$ Faculty of Health Sciences, The University of Sydney, Sydney, New South Wales, Australia \\ ${ }^{2}$ The George Institute for Global Health \& Institute of Bone and Joint Research/The Kolling Institute, Sydney Medical \\ School, The University of Sydney, Sydney, Australia \\ ${ }^{3}$ Centre for Epidemiology \& Biostatistics, Melbourne School of Population and Global Health, The University of \\ Melbourne, Melbourne, Victoria, Australia \\ ${ }^{4}$ The George Institute for Global Health, Sydney Medical School, The University of Sydney, Sydney, New South Wales, \\ Australia \\ ${ }^{5}$ Center for Muscle and Joint Health, University of Southern Denmark and Nordic Institute of Chiropractic and Clinical \\ Biomechanics, Odense, Denmark \\ ${ }^{6}$ Department of General Practice, Erasmus MC, University Medical Centre, Rotterdam, Netherlands \\ ${ }^{7}$ Neuroscience Research Australia and The University of New South Wales, Sydney, New South Wales, Australia
}

\begin{abstract}
Low back pain (LBP) is a major health problem globally, but approaches to prevention are not yet clearly identified because modifiable risk factors are not well established. Although physical activity is one promising modifiable risk factor, it is still not known what types and doses of physical activity are protective or harmful for LBP. The aim of this study is to establish the feasibility of a definitive cohort study that will investigate the effects of different types and doses of physical activity on the risk of developing recurrent LBP while accounting for genetic factors. This will be a pilot longitudinal twin study and twins will be recruited from the Australian Twin Registry, and will be followed over 1 year. Thirty adult complete twin pairs with a history of LBP, but symptom free at recruitment, will be included. Data on physical activity (predictor) will be collected using four questionnaires and an objective measure (accelerometer) at baseline and at 6-month follow-up. Twins will also complete an additional physical activity questionnaire monthly. Data on LBP (outcome) will be collected at baseline and weekly. Data will be collected using short message service (SMS) and email. We will keep records of the recruitment rate, follow-up rate, and completeness of data. Barriers to completing the study will be investigated. The results of this study will inform the design and implementation of a future definitive study, which will help to clarify the effects of different types and doses of physical activity on the risk of developing recurrent LBP.
\end{abstract}

Keywords: low back pain, physical activity, twin study, longitudinal study, risk factors

The latest Global Burden of Diseases Study ranked low back pain (LBP) as the highest contributor to disability globally, expressed as years of life lived with disability (YLD; Global Burden of Disease Study, 2015). LBP is also a costly condition, with total treatment costs estimated at $\$ 4.8$ billion a year in Australia (Arthritis and Osteoporosis Victoria, 2013). Implementing preventive strategies for LBP would be a logical method to reduce the burden of this condition on people's lives and on society.

Although many preventive strategies for LBP have been proposed, most have failed to substantially reduce the incidence and recurrence rates of LBP (Steffens et al., 2016).
A plausible explanation for the lack of effectiveness of these programs is our poor understanding of the causes of this condition, and therefore identifying strong modifiable risk factors is essential. Importantly, most previous studies investigating risk factors for LBP have not considered the

RECEIVED 27 May 2016; ACCEPTED 7 July 2016

ADDRESS FOR CORRESPONDENCE: Marina B. Pinheiro, Faculty of Health Sciences, The University of Sydney, 75 East Street, Lidcombe, Sydney NSW 2141, Australia. E-mail: mdeb9852@uni.sydney.edu.au 
influence of genetic factors, which have been shown to explain up to $67 \%$ of the underlying disposition to develop LBP (Ferreira et al., 2013; Nielsen et al., 2012).

A recent review has found moderate quality evidence that exercise in combination with education decreases the risk of LBP by half (Steffens et al., 2016). However, given the limited number of trials identified in the literature, the specific effects of type, and dosage of physical activity or exercise on the risk of LBP could not be estimated in this review. This is particularly unfortunate because various forms of physical activity are associated with a range of health benefits (Pedersen \& Saltin, 2015). Recent systematic reviews have shown that the association between physical activity and LBP in the general population is conflicting (Heneweer et al., 2011; Sitthipornvorakul et al., 2011). This may be because most studies have focused on single types of physical activity (e.g., lifting or standing at the worksite, engagement in physical activity during leisure time) rather than a comprehensive assessment of physical activity across all relevant domains. Different types of physical activity seem to have a different effect on the risk of LBP. For instance, heavy workload and frequency of lifts were found to increase the risk of developing LBP, whereas engagement in leisure physical activity has been shown to reduce its risk (Holtermann et al., 2012). Additionally, it is plausible that the association between physical activity and LBP depends not only on the type of physical activity, but also on its duration, frequency, and intensity. For instance, a previous study has found that heavy domestic physical activity (vigorous gardening or heavy work around the house) is associated with increased risk of LBP (Hübscher et al., 2014; Steffens et al., 2015), while other studies have shown that moderate gardening or yard work is associated with decreased risks for LBP (Heneweer et al., 2011).

There were many limitations in previous studies that are contributing to the uncertainty in this field. First, previous investigations have failed to differentiate the types and doses of physical activity. Second, the majority of studies measured physical activity through self-reported questionnaires only. In the two most recent systematic reviews in the field (Heneweer et al., 2011; Sitthipornvorakul et al., 2011), only one study used an objective measure (accelerometer) of physical activity and it has been shown that self-reported measures can overestimate engagement in physical activity (Lee et al., 2011) and are overall not strongly associated with objective assessments of physical activity (Wedderkopp et al., 2003). Finally, genetic factors have not been accounted for in the LBP-physical activity-relationship; which is an important omission, as genetic factors have been shown to have a significant impact on both LBP (Ferreira et al., 2013) and physical activity engagement (Santos et al., 2012). For example, a previous study conducted by our group found that the relationship between physical activity and LBP changes once genetic factors are considered (Hübscher et al., 2014) because engagement in do- mestic physical activity was associated with higher prevalence of $\mathrm{LBP}(\mathrm{OR}=1.63,95 \% \mathrm{CI} 1.04$ to 2.54$)$ and this result was more noticeable in the case-control analysis where the genetic factor was adjusted for ( $\mathrm{OR}=2.88,95 \% \mathrm{CI}$ to 1.29-6.43).

The effects of physical activity on LBP would be best elucidated if risk studies included a comprehensive assessment of types and dosages of physical activity, including the use of an objective measure and addressed genetic influences. The aim of this study is therefore to establish the feasibility of a definitive cohort study that will investigate the effects of different types (domestic, recreational, and work-related) and doses (frequency, duration, intensity - i.e., sedentary, light, moderate, and vigorous) of physical activity on the risk of recurrent LBP while accounting for genetic factors. Specifically, we aim to determine the rate of recruitment of adult twins registered at the Australian Twin Registry (ATR) to calculate sample size requirements for the definitive study, estimate the follow-up rate and compliance, assess completeness of data collection, and identify possible barriers that could be experienced during the implementation of the study.

\section{Methods}

\section{Study Design}

This is a feasibility longitudinal twin cohort study investigating the effects of physical activity on LBP. All data collection procedures will be conducted remotely (i.e., SMS, e-mail, telephone, accelerometer). This study has been approved by the ATR and the University of Sydney Human Research Ethics Committee (Project No: 2015/407).

\section{Participants}

Participants will be recruited from the ATR (Figure 1). Thirty complete twin pairs will be included and will be followed for 1 year. Twin pairs will be included if both twins within the pair: are aged 18 years or older; have current internet access, an active e-mail account, and a smartphone with internet access; agree to be included in the study; and have a history of LBP, but have been symptom-free (pain intensity $<2$ on a $0-10$ pain scale) for at least 4 weeks at recruitment. There will be no restriction on twins' zygosity, gender, or other demographic variables. Participants will be excluded if they are pregnant or if they have known or suspected serious spinal pathology (metastatic, inflammatory, or infective diseases of the spine) or a history of spinal surgery in the last 12 months.

\section{Recruitment}

Twins registered with the ATR will be invited to participate in this study. Complete twin pairs meeting all inclusion criteria will be invited to participate in the study and will be included if both twin within a pair provide written informed consent. 


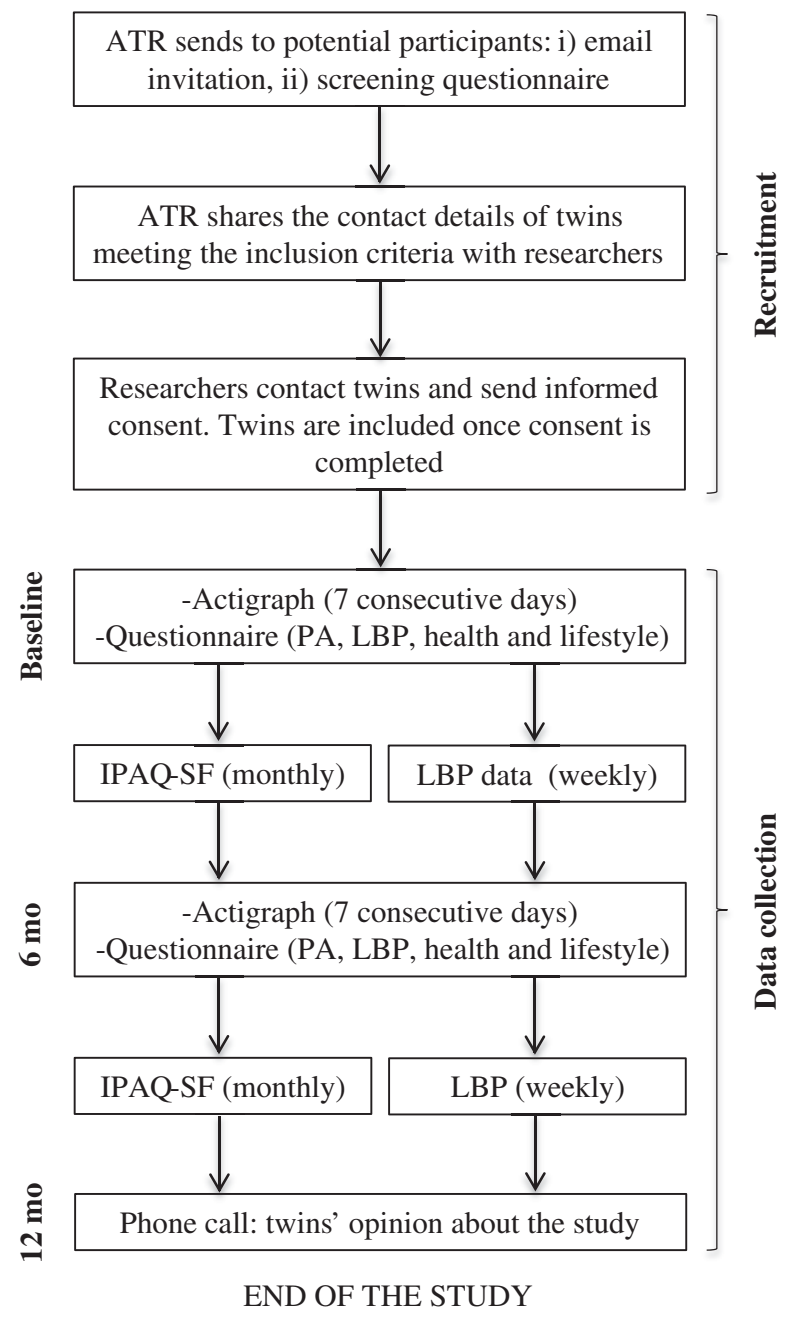

\section{FIGURE 1}

Flow diagram of the study protocol.

Note: ATR = Australian Twin Registry; LBP = low back pain; PA = physical activity; mo $=$ months; IPAQ-SF $=$ International Physical Activity Questionnaire - Short Form.

\section{Predictors and Outcomes Assessment}

Assessment of predictors. Five physical activity measures will be assessed as potential predictors of LBP. Physical activity is usually described by type (domestic, transportation, recreational, work-related) and dose (frequency, duration, intensity; Heneweer et al., 2009). In our study, we will use five measures, one objective and four self-reported measures, to provide a comprehensive assessment of physical activity to capture all types and consider day-to-day variation (Table 1; Warren et al., 2010).

Objective assessment of physical activity. We will use accelerometers (Actigraph GT1M/GT3X) to objectively assess the participant's physical activity engagement. The use of accelerometers, such as the Actigraph GT1M/GT3X, has been suggested to offer the most accurate, sensitive, and valid measure of physical activity (Warren et al., 2010b).
Actigraph GT1M/GT3X are devices that assess body movements (acceleration) and records the number of activity counts summed and provides a measure of overall physical activity (mean counts per minute; CPM) and intensity specific (sedentary, light, moderate, vigorous) physical activity (Chen \& Bassett, 2005). The assessment of physical activity using the Actigraph GT1M/GT3X accelerometer will be performed in accordance with best practice recommendations for using physical activity monitors in populationbased research (Tully et al., 2014; Warren et al., 2010). Participants will be required to wear the device for seven consecutive days during waking hours (before answering the baseline questionnaire) on the right hip, attached with an adjustable elastic belt. Participants will be instructed to remove the device during water-based activities or bathing. Data will be included if the participant accumulated at least 10 hours of valid activity recordings per day for at least 4 days. 'Non-wear' time will be determined according to previously established algorithm (Choi et al., 2011).

Self-reported assessment of physical activity. To complement the assessment of different types of physical activity, we will use the long and short form of the International Physical Activity Questionnaire (IPAQ; Hagstromer et al., 2006; Lee et al., 2011), the Physical Workload Index questionnaire (Hollmann et al., 1999), and the Recent Physical Activity questionnaire (RPAQ; Besson et al., 2010). The IPAQ has acceptable measurement properties (Hagstromer et al., 2006) and assesses frequency and duration of physical activity in five different domains (i.e., occupation, transportation, domestic work, recreation, sedentary activity; Hagstromer et al., 2006). We will measure physical activity at work using the Physical Workload Index questionnaire (Hollmann et al., 1999), a reliable and valid instrument to assess the frequency of people's engagement in different postures and tasks while at work. The RPAQ has acceptable measurement properties and assesses frequency and duration of sports-related physical activity (Besson et al., 2010).

Assessment of outcome. The primary outcome will be the number of days of LBP with a pain intensity of $>2$ on an 11point numerical rating scale (Childs et al., 2005) over the 1year period (Stanton et al., 2011), provided that these were preceded by a period of at least 1 month without LBP with an intensity of $>2$ on a $0-10$ pain scale. The secondary outcomes for all participants will be: (1) number of days of activity limitation associated with LBP over the 1-year period; (2) number of days of care-seeking associated with LBP over the 1-year period; (3) number of days of medication use for LBP over the 1-year period. The following definition of LBP will be used: 'pain in the area between the 12th rib and buttock crease with or without leg pain' (de Vet et al., 2002; Henschke et al., 2008). 
TABLE 1

Description of Measures of Physical Activity

\begin{tabular}{|c|c|c|c|}
\hline Method & Type of physical activity & Dose of physical activity & Time point \\
\hline Actigraph (Accelerometer) & Habitual/daily physical activity & Frequency, duration, intensity & Baseline and 6-month \\
\hline IPAQ long version & $\begin{array}{l}\text { Work related, domestic, } \\
\text { transportation, recreational }\end{array}$ & Frequency, duration & Baseline and 6-month \\
\hline IPAQ short version & Daily physical activity & Frequency, duration & $\begin{array}{l}\text { Monthly, except at baseline } \\
\text { and 6-month }\end{array}$ \\
\hline Physical Workload Index & Work related & Frequency & Baseline and 6-month \\
\hline Recent physical activity & Sports related & Frequency, duration & Baseline and 6-month \\
\hline
\end{tabular}

Note: IPAQ = International Physical Activity Questionnaire.

Other variables. We will also collect anthropometric information, data on depression, sleep quality, and smoking as these variables have been reported to affect LBP and physical activity engagement. Participants will be required to report their height and weight and they will measure their waist and hip circumference. We will send the participants a measuring tape and instructions by post (Ayala et al., 2014). The Pittsburgh Sleep Quality Index (PSQI) will be used to assess the subjective sleep quality of the participants (Buysse et al., 1989). The PSQI is an 18-item selfreport questionnaire and Assesses sleep disturbances in the last month in seven domains: subjective sleep quality, sleep latency (the time it takes to fall asleep), sleep duration, habitual sleep efficiency (the ratio of total sleep time to time spent in bed), sleep disturbances, use of sleeping medication, and daytime dysfunction. The total score is composed of the sum of scores for these seven domains and can range from 0 to 21, with higher scores meaning worse sleep quality and a total score of 5 or more being indicative of poor sleep quality. The short form of the Depression Anxiety Stress Scales (DASS-21) will be used to assess symptoms of depression, anxiety, and stress. The DASS- 21 is a quantitative measure of distress along the three axes of depression, anxiety, and stress. It is composed of 21 items. The cut-off for severe or extremely severe for symptoms of depression is score $>10$, for anxiety is score $>7$, and for stress is score $>12$ (Henry \& Crawford, 2005). Participants will also be asked about their smoking habits. Additional information, such as data on alcohol consumption, general health, and co-morbidities are also going to be collected.

\section{Procedures}

Baseline assessment. First, at baseline, the Actigraph will be sent by post to participants with a package containing detailed instructions on placement and wearing time, a physical activity diary, a prepaid envelope to return the equipment, detailed instructions on how to return the equipment, and contact details of study personnel.

Twins will be asked to complete a daily physical activity diary and to detail daily wearing and non-wearing times during the 7-day period. To ensure compliance, participants will be sent two short message service (SMS) reminders (on the third and fifth day of the 7-day period). At the end of the 7-day period, twins will be required to mail the device to study investigators. In case participants fail to meet the minimum wear time, we will attempt to send the Actigraph to participants again.

The baseline questionnaire will be completed at the end of the 7-day period of use of the Actigraph with a one week recall period so that all measures of physical activity are timely synchronized. To answer the baseline questionnaire, twins will be asked to follow a questionnaire link sent via email. The questionnaire will include questions on anthropometric data, LBP, self-reported physical activity (all three questionnaires), depression (DASS-21), sleep quality (PSQI), and smoking.

Follow-up assessment. In order to assess changes in physical activity levels from baseline, twins will be required to answer every month the IPAQ-SF (Lee et al., 2011). An SMS or an email with a link to the questionnaire will be sent to participant twins. Additionally, after 6 months of study entry, participating twins will be required to wear the Actigraph again and to answer the three self-reported physical activity questionnaires. The same procedures used for the baseline assessment will be employed.

During the whole study duration, participants will be required to answer weekly questions about LBP, including intensity, activity limitation, care-seeking, and medication use associated with LBP (Appendix A). Participants will receive an SMS or email with a link to the questions. Twins will be contacted by the researchers if they do not respond to the questionnaire for four consecutive weeks.

\section{Feasibility Investigation}

Measurement of recruitment. Throughout the recruitment process, records will be kept regarding the number of twins that answered the screening questionnaire. If the twins are not admitted to the study, the reason why they were ineligible for inclusion will be recorded. Similarly, if eligible, the reasons declining participation in the study will be noted.

Measurement of follow-up rate, compliance, and completeness of data collection. The number of participants completing the study and answering the follow-up questionnaires will be recorded. We will also record the 
compliance with the Actigraph and with answering the follow-up questions, as well as the number of follow-ups answered by each participant. The number of participants lost in each phase of the study will be noted and the reasons for dropping out will be recorded when possible. If a participant decides to drop out from the study, he/she will be sent an SMS or email (according to the preferred method of contact) asking about their reasons for dropping out (Appendix B). We specifically aim to investigate if twins decided to drop out as a result of the method of data collection or frequency of contact messages.

Difficulties/barriers to completing the study. Every issue experienced during the study will be recorded, such as technical problems. This information will help us to anticipate the potential problems when conducting the full study in the future. Those participants who complete the study will be contacted by phone and they will be asked about their experience, difficulties, and barriers encountered during the study. A semi-structured interview will be used and it will be recorded.

Analysis. All data will be exported to a statistical software package and will be cleaned and checked for errors. The success of our recruitment strategies will be measured by summarizing number of twins approached, screened, eligible, and who provided consent. Additionally, monthly recruitment rates will be computed. This data will be used to determine the recruitment period and number of twins that should be approached to achieve the final sample size.

We will assess the acceptability of the tools used to collect data by analyzing the level of missing data for each of the predictors and outcome measures and follow-up rate. Descriptive statistics will be used to analyze the data on reasons for dropping out.

The data gathered by the phone interviews will be analyzed to investigate barriers and facilitators to completing the study. The main interview areas to be explored will be the effect of physical activity on LBP, reasons for participation in the study, their interpretation of study information and documentation, their views on the different methods used to collect data, and barriers/motivators to participate in the study.

Actigraphs' data will be processed using the manufacturer's software (Actlife) that will be used to initialize the devices for data recording and also for downloading the data. Activity files will be screened for compliance and quality and will be manually checked against participants' diaries to verify wear time, and any erroneous data will be deleted. Actigraph activity data will then be exported into a spreadsheet with output including overall physical activity (minutes/day), time spent (minutes/day) in sedentary, light, moderate, and vigorous physical activity, and energy expenditure.
Descriptive analysis will be performed for the predictors and outcome measures. As this is a feasibility study, analysis will focus on confidence interval estimation (rather than hypothesis testing) and will help us to determine sample size requirement for the full study, based on the standard error of the estimate of the correlation between LBP and physical activity.

Regarding the feasibility outcomes, any of the following would suggest that the definitive study is not feasible in the current format and might require changes: follow-up rate $<80 \%$, recruitment rate $<10 \%$ (considering that the ATR currently has over 35,000 twin pairs registered), $<80 \%$ of valid data on predictors and outcomes.

\section{Discussion}

This article details the rationale and protocol of a pilot longitudinal twin cohort study to investigate the effects of different types and doses of physical activity on LBP. Although the role of physical activity in the prevention of several noncommunicable diseases is clear (Pedersen \& Saltin, 2015), this is not the case in LBP. It is likely that appropriate engagement in physical activity is helpful in LBP but it is still not known what type(s) and dose(s) of physical activity are beneficial. The question of the type and dose of physical activities that increase risk or protect against LBP remains largely unresolved, and sensibly advising patients is very difficult. This is unclear because previous studies have mostly used self-reported measures of physical activity and have not addressed the effects of genetics on this association.

Our study will address these issues and further our understanding of the type and dose of physical activity that is beneficial or harmful in this population. Although the employment of an objective measure of physical activity represents an advance in the field in relation to previous studies, the Actigraph does not capture all kinds of physical activity. Some aspects of physical activity, such as loads, angles, and time spent in different body postures (lying, sitting, standing, bicycling) will remain not investigated. Additionally, Actigraph is not waterproof. However, this information not captured by the Actigraph will be supplemented by the questionnaires.

As this study has innovative features, such as the data collection method and the employment of a twin design, it is imperative to conduct a pilot study to ensure every part of the study will be successfully conducted in the full study. The results of the feasibility study will be presented as soon as they are available and will inform the planning and implementation of the future larger cohort study.

\section{Acknowledgments}

MBP holds the International Post-graduate Research Scholarship/Post-graduate Award from the Australian Government. MLF is a Sydney Medical Foundation Fellow, The 
University of Sydney. CGM's research fellowship is funded by the National Health and Medical Research Council of Australia.

\section{Financial Support}

This research received competitive funding form the University of Sydney Bridging Support Grant - NHMRC Project Grant: APP1086738.

\section{Conflicts of Interests}

None.

\section{Ethical Standards}

The authors assert that all procedures contributing to this work comply with the ethical standard of the relevant national and institutional committees on human experimentation and with the Helsinki Declaration of 1975, as revised in 2008 .

\section{References}

Arthritis and Osteoporosis Victoria. (2013). A problem worth solving. The rising cost of musculoskeletal conditions in Australia. Melbourne, Australia: Arthritis and Osteoporosis Victoria.

Ayala, A. M. C., Nijpels, G., \& Lakerveld, J. (2014). Validity of self-measured waist circumference in adults at risk of type 2 diabetes and cardiovascular disease. BMC Medicine, 12, 170.

Besson, H., Brage, S., Jakes, R. W., Ekelund, U., \& Wareham, N. J. (2010). Estimating physical activity energy expenditure, sedentary time, and physical activity intensity by selfreport in adults. American Journal of Clinical Nutrition, 91, 106-114.

Buysse, D. J., Reynolds, C. F., Monk, T. H., Berman, S. R., \& Kupfer, D. J. (1989). The Pittsburgh Sleep Quality Index: A new instrument for psychiatric practice and research. Psychiatry Research, 28, 193-213.

Chen, K. Y., \& Bassett, D. R. Jr. (2005). The technology of accelerometry-based activity monitors: Current and future. Medicine and Science in Sports and Exercise, 37, S490-S500.

Childs, J. D., Piva, S. R., \& Fritz, J. M. (2005). Responsiveness of the numeric pain rating scale in patients with low back pain. Spine (Phila Pa 1976), 30, 1331-1334.

Choi, L., Liu, Z. W., Matthews, C. E., \& Buchowski, M. S. (2011). Validation of accelerometer wear and nonwear time classification algorithm. Medicine and Science in Sports and Exercise, 43, 357-364.

de Vet, H. C., Heymans, M. W., Dunn, K. M., Pope, D. P., van der Beek, A. J., Macfarlane, G. J., ... Croft, P. R. (2002). Episodes of low back pain: A proposal for uniform definitions to be used in research. Spine (Phila Pa 1976), 27, 2409-2416.

Ferreira, P. H., Beckenkamp, P., Maher, C. G., Hopper, J. L., \& Ferreira, M. L. (2013). Nature or nurture in low back pain? Results of a systematic review of studies based on twin samples. European Journal of Pain, 17, 957-971.
Global Burden of Disease Study, C. (2015). Global, regional, and national incidence, prevalence, and years lived with disability for 301 acute and chronic diseases and injuries in 188 countries, 1990-2013: A systematic analysis for the global burden of disease study 2013. Lancet, 386, 743-800.

Hagstromer, M., Oja, P., \& Sjostrom, M. (2006). The international physical activity questionnaire (IPAQ): A study of concurrent and construct validity. Public Health Nutrition, 9, 755-762.

Heneweer, H., Staes, F., Aufdemkampe, G., van Rijn, M., \& Vanhees, L. (2011). Physical activity and low back pain: A systematic review of recent literature. European Spine Journal, 20, 826-845.

Heneweer, H., Vanhees, L., \& Picavet, H. S. (2009). Physical activity and low back pain: A U-shaped relation? Pain, 143, 21-25.

Henry, J. D., \& Crawford, J. R. (2005). The short-form version of the Depression Anxiety Stress Scales (DASS-21): Construct validity and normative data in a large nonclinical sample. British Journal of Clinical Psychology, 44, 227-239.

Henschke, N., Maher, C. G., Refshauge, K. M., Herbert, R. D., Cumming, R. G., Bleasel, J., ... McAuley, J. H. (2008). Prognosis in patients with recent onset low back pain in Australian primary care: Inception cohort study. British Medical Journal, 337, a171.

Hollmann, S., Klimmer, F., Schmidt, K. H., \& Kylian, H. (1999). Validation of a questionnaire for assessing physical work load. Scandinavian Journal of Work, Environment \& Health, 25, 105-114.

Holtermann, A., Hansen, J. V., Burr, H., Sogaard, K., \& Sjogaard, G. (2012). The health paradox of occupational and leisure-time physical activity. British Journal of Sports Medicine, 46, 291-295.

Hübscher, M., Ferreira, M. L., Junqueira, D. R., Refshauge, K. M., Maher, C. G., Hopper, J. L., \& Ferreira, P. H. (2014). Heavy domestic, but not recreational, physical activity is associated with low back pain: Australian Twin low BACK pain (AUTBACK) study. European Spine Journal, 23, 20832089.

Lee, P. H., Macfarlane, D. J., Lam, T. H., \& Stewart, S. M. (2011). Validity of the international physical activity questionnaire short form (IPAQ-SF): A systematic review. International Journal of Behavioral Nutrition and Physical Activity, $8,115$.

Nielsen, C. S., Knudsen, G. P., \& Steingrimsdottir, O. A. (2012). Twin studies of pain. Clinical Genetics, 82, $331-340$

Pedersen, B. K., \& Saltin, B. (2015). Exercise as medicine - Evidence for prescribing exercise as therapy in 26 different chronic diseases. Scandinavian Journal of Medicine \& Science in Sports, 25, 1-72.

Santos, D. M. D. E., Katzmarzyk, P. T., Seabra, A. F. T., \& Maia, J. A. R. (2012). Genetics of physical activity and physical inactivity in humans. Behavior Genetics, 42, 559-578.

Sitthipornvorakul, E., Janwantanakul, P., Purepong, N., Pensri, P., \& van der Beek, A. J. (2011). The association between 
physical activity and neck and low back pain: A systematic review. European Spine Journal, 20, 677-689.

Stanton, T. R., Latimer, J., Maher, C. G., \& Hancock, M. J. (2011). A modified Delphi approach to standardize low back pain recurrence terminology. European Spine Journal, 20, 744-752.

Steffens, D., Ferreira, M. L., Latimer, J., Ferreira, P. H., Koes, B. W., Blyth, F., ... Maher, C. G. (2015). What triggers an episode of acute low back pain? A case-crossover study. Arthritis Care \& Research, 67, 403-410.

Steffens, D., Maher, C. G., Pereira, L. S., Stevens, M. L., Oliveira, V. C., Chapple, M., ... Hancock, M. J. (2016). Prevention of low back pain: A systematic review and metaanalysis. JAMA Internal Medicine, 176, 199-208.

Tully, M. A., McBride, C., Heron, L., \& Hunter, R. F. (2014). The validation of Fitbit Zip physical activity monitor as a measure of free-living physical activity. BMC Research Notes, 7, 952.

Warren, J. M., Ekelund, U., Besson, H., Mezzani, A., Geladas, N., Vanhees, L., \& Experts Panel (2010). Assessment of physical activity - A review of methodologies with reference to epidemiological research: A report of the exercise physiology section of the European association of cardiovascular prevention and rehabilitation. European Journal of Cardiovascular Prevention and Rehabilitation, 17, 127-139.

Wedderkopp, N., Leboeuf-Yde, C., Andersen, L. B., Froberg, K., \& Hansen, H. S. (2003). Back pain in children - No association with objectively measured level of physical activity. Spine, 28, 2019-2024.

\section{Appendix A}

\section{Low Back Pain Weekly Questions}

1. Have you had low back pain in the last 7 days?

( ) Yes

( ) No

Observation: the following questions will only appear if the participant has answered 'Yes' for the previous question.

2. For how many days have you had low back pain in the last 7 days?
( ) 1
( ) 2
( ) 3
( ) 4
( ) 5
() 6
() 7

3. Please indicate on average what was the intensity of your low back pain on a scale from 0 to 10 , where 0 means 'no pain' and 10 means 'the worst pain imaginable', over the last 7 days.

\begin{tabular}{lccccccccccc}
\hline 0 & 1 & 2 & 3 & 4 & 5 & 6 & 7 & 8 & 9 & 10 \\
\hline $\begin{array}{l}\text { No } \\
\text { pain }\end{array}$ & & & & & $\begin{array}{l}\text { Moderate } \\
\text { pain }\end{array}$ & & & & & $\begin{array}{l}\text { Worst } \\
\text { possible } \\
\text { pain }\end{array}$ \\
\hline
\end{tabular}

4. Was the low back pain bad enough to limit your usual activities in the last 7 days?

( ) Yes (the following options will only appear if they answer 'yes')

( ) No (go to next question)

( ) Work

( ) Socializing

( ) Sports

( ) Hobbies

( ) Intimacy

( ) Chores

( ) I did not have any activities limited because of my pain

For how many days was the low back pain bad enough to limit your activities? (this question will appear for each option selected by the participant)
( ) 1
( ) 2
( ) 3
() 4
( ) 5
() 6
( ) 7

5. Have you sought any treatment for this low back pain in the last 7 days?

( ) Yes (the following options will only appear if they answer 'yes')

( ) No (go to next question)

( ) GP

( ) Physiotherapist

( ) Chiropractor

( ) Emergency department

( ) Surgical procedure

( ) Other Please specify:

I have treated myself with (does not include medication use/select as many as applicable):

( ) heat pack

( ) bed rest

( ) light exercise, such as walking

( ) hot shower

( ) by seeking information on internet and books.

( ) None of the mentioned above

( ) I did not seek any treatment for my pain

For how many days did you have to seek treatment for your low back pain? (this question will appear for each option selected by the participant)

( ) 1

( ) 2

() 3

( ) 4

( ) 5

() 6

( ) 7 
6. Have you taken any medication for your low back pain in the last 7 days?

( ) Yes (the following options will only appear if they answer 'yes')

( ) No (go to next question)

( ) Non-Opioid Analgesics (Examples: Paracetamol, Aspirin, Nurofen, Voltaren)

( ) Weak Opioid Analgesics (Examples: Codeine, Oxycodone, Tramadol)

( ) Strong Opioid (Example: Morphine)

( ) Antidepressants (Examples: Zolof, Prozac, Efexor, Allegron, Avanza, Edronax)

( ) Natural pain relievers

( ) Others Please specify:

( ) I did not take any medication for my pain.

For how many days did you have to take medication for your low back pain? (this ques- tion will appear for each option selected by the participant)
( ) 1
( ) 2
( ) 3
( ) 4
( ) 5
( ) 6
( ) 7

\section{Appendix B}

\section{Reasons for Dropping Out}

Why did you decide to drop out of this study?

1. Method of data collection (short message service SMS).

2. Repetitiveness of SMS

3. Frequency of SMS.

4. Other. Please specify: 